OPEN ACCESS

Edited by:

Zhulong Chan,

Huazhong Agricultural University,

China

Reviewed by:

Venkateswara Rao Khareedu,

Osmania University, India

Bishal Gole Tamang,

University of Minnesota, USA

*Correspondence:

Chengwei $L i$

lichengweiwau@hotmail.com

tThese authors have contributed equally to this work.

Specialty section: This article was submitted to

Plant Abiotic Stress,

a section of the journa

Frontiers in Plant Science

Received: 24 November 2016

Accepted: 20 February 2017

Published: 06 March 2017

Citation

Tang Y, Liu K, Zhang J, Li X, Xu K, Zhang $Y$, Qi J, Yu D, Wang J and Li C (2017) JCDREB2, a Physic Nut AP2/ERF Gene, Alters Plant Growth and Salinity Stress Responses in Transgenic Rice.

Front. Plant Sci. 8:306. doi: 10.3389/fpls.2017.00306

\title{
JcDREB2, a Physic Nut AP2/ERF Gene, Alters Plant Growth and Salinity Stress Responses in Transgenic Rice
}

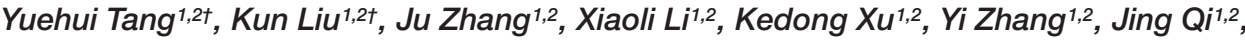
Deshui Yu', , Jian Wang ${ }^{1,2}$ and Chengwei Li,2* $^{1,2}$
\end{abstract}

1 Henan Key Laboratory of Crop Molecular Breeding and Bioreactor, Zhoukou, China, ${ }^{2}$ Key Laboratory of Plant Genetics and Molecular Breeding, Zhoukou Normal University, Zhoukou, China

Transcription factors of the AP2/ERF family play important roles in plant growth, development, and responses to biotic and abiotic stresses. In this study, a physic nut AP2/ERF gene, JCDREB2, was functionally characterized. Real-time PCR analysis revealed that JCDREB2 was expressed mainly in the leaf and could be induced by abscisic acid but suppressed by gibberellin (GA) and salt. Transient expression of a JcDREB2-YFP fusion protein in Arabidopsis protoplasts cells suggested that JcDREB2 is localized in the nucleus. Rice plants overexpressing JCDREB2 exhibited dwarf and GA-deficient phenotypes with shorter shoots and roots than those of wild-type plants. The dwarfism phenotype could be rescued by the application of exogenous $\mathrm{GA}_{3}$. The expression levels of GA biosynthetic genes including OsGA20ox1, OsGA20ox2, OsGA20ox4, OsGA3ox2, OsCPS1, OsKO2, and OsKAO were significantly reduced in plants overexpressing JCDREB2. Overexpression of JCDREB2 in rice increased sensitivity to salt stress. Increases in the expression levels of several salt-tolerancerelated genes in response to salt stress were impaired in JCDREB2-overexpressing plants. These results demonstrated not only that JCDREB2 influences GA metabolism, but also that it can participate in the regulation of the salt stress response in rice.

Keywords: AP2/ERF transcription factor, physic nut (Jatropha curcas L.), salinity, gibberellin, rice

\section{INTRODUCTION}

Abiotic stresses, such as high salinity and drought, adversely affect plant growth and agricultural productivity, resulting in serious losses in yield. To cope with these adverse conditions, plants have evolved various defense mechanisms enabling them to tolerate environmental stresses. Transcription factors are significant candidates for mediating plant responses to stress tolerance because of their roles as master regulators of many stress-responsive genes (Singh et al., 2002). In the past few years, many transcription factors belonging to the AP2/ERF, NAC, MYB, WRKY, bHLH, and NF-Y families have been characterized and shown to play significant roles in plant 
responses to biotic and abiotic stresses (Li et al., 2010; Jiang et al., 2014; Yan et al., 2014; Chen M. et al., 2015; Shao et al., 2015; Zhang et al., 2015; Hong et al., 2016; Tang et al., 2016).

The AP2/ERF transcription factors are defined by a conserved AP2/ERF domain which consists of about 60 to 70 amino acids, and they can be divided into four subfamilies, AP2, ERF, DREB, RAV and Soloist, depending on the number of AP2/ERF domains they contain and the presence or absence of other DNA binding domains (Sakuma et al., 2002; Wessler, 2005). Recently there has been increasing evidence that the AP2/ERF family proteins are involved in the responses of plants to biotic and abiotic stresses. For instance, DREB transcription factor proteins bind to the DRE/CRT (drought responsive/C-Repeat) cis-acting elements (core motif: G/ACCGAC) and control the expression of many stressresponsive genes (Shinozaki and Yamaguchi-Shinozaki, 2000). In rice and Arabidopsis, DREB transcriptional activators such as those encoded by OsDREB2A, DREB2C, and AtDREB1A have been isolated. OsDREB2A expression is induced by drought, low-temperature and salt stresses, and overexpression of OsDREB2A significantly affects salt tolerance by altering the accumulation of osmolytes, such as soluble sugars and free proline, in transgenic soybean (Zhang et al., 2012). Overexpression of DREB2C and AtDREB1A confers tolerance to, respectively, salt and drought in transgenic plants (Song et al., 2014; Wei et al., 2016). DREB-encoding genes in several other species, genes which include $V v D R E B 2 A, S s D R E B$, and $G m D R E B 1$, have been identified as playing significant parts in salinity tolerance through regulating the expression of stress-responsive genes and physiological processes (Jin et al., 2010; Zhang et al., 2014; Chen H. et al., 2015). DREB transcription factors are also involved in plant growth and development. Overexpression of TINY, a DREB subfamily gene, results in a dwarf phenotype in Arabidopsis under normal growth conditions (Sun et al., 2008). Although many DREB transcription factors have been extensively studied in various plants, further efforts are still needed to identify other novel DREB genes that are involved in plant development and stress responses.

Physic nut (Jatropha curcas L.) is a multipurpose perennial woody plant belonging to the Euphorbiaceae family. Its abilities to grow easily in barren soil and endure drought and salinity, its adaptation to a wide range of agro-climatic conditions, and its high seed oil content, mean that the physic nut has emerged as a promising source of biodiesel (Openshaw, 2000). Our previous study identified 119 putative AP2/ERF genes in the physic nut genome, and we observed that a gene (JCGZ_24071) which we designated JcDREB2 was strongly repressed by salt stress (Tang et al., 2016). JcDREB2 was therefore chosen for further functional analysis. In the present study, we demonstrate that JCDREB2 encodes a transcription factor whose expression is regulated by salinity, gibberellic acid $\left(\mathrm{GA}_{3}\right)$ and abscisic acid (ABA) treatments. Our results indicated that overexpression of JCDREB2 in rice resulted in both a dwarf phenotype, via downregulation of key genes involved in GA biosynthesis, and increased sensitivity to salinity stress.

\section{MATERIALS AND METHODS}

\section{Plant Materials}

Jatropha curcas seeds were collected from Guizhou province, China and planted on farm land in Guangzhou, Guangdong province, China. For analysis of JcDREB2 gene expression in physic nut, roots, stem cortex, leaves, flowers, and seeds were sampled at 35 days after pollination and stored at $-80^{\circ} \mathrm{C}$ until required for RNA isolation. For salinity treatment, plants at the six-leaf stage were irrigated with Hoagland solution plus $150 \mathrm{mM}$ $\mathrm{NaCl}$. For $\mathrm{GA}_{3}$ and $\mathrm{ABA}$ treatments, plants at the six-leaf stage were sprayed with $100 \mu \mathrm{M} \mathrm{GA}_{3}, 100 \mu \mathrm{M}$ ABA or distilled water (control), and the fourth leaves were collected after 1, 3, 6, and $12 \mathrm{~h}$ of $\mathrm{GA}_{3}$ and ABA stresses.

The japonica rice (Oryza sativa L.) cv. Zhonghua 11 (ZH11) was used as the wild type in this study. Seeds were germinated and cultured in soil in basins in a greenhouse under natural sunlight.

\section{Protein Sequence and Phylogenetic Analyses}

All protein sequences of JcDREB2 orthologs were downloaded from GenBank ${ }^{1}$. ClustalX was used to analyze multiple sequence alignment (Thompson et al., 1997). The phylogenetic relationship of JcDREB2 orthologs was constructed, using MEGA 5 software, by the Neighbor-Joining method with 1000 bootstrap replicates (Tamura et al., 2011).

\section{Subcellular Localization}

The full length coding domain sequence of JcDREB2 (after removal of the termination codon) was amplified by RTPCR from total RNA extracted from physic nut leaves using the primers given in Supplementary Table S1. The target sequence was cloned into the pSAT6-eYFP-N1 vector to construct the pSAT6-JcDREB2-eYFP fusion expression vector. The 35S::JcDREB2-YFP construct and a control (35S::YFP) plasmid were introduced into Arabidopsis protoplasts by the polyethylene glycol (PEG) mediated method. Next the transformed cells were incubated in the light for $16 \mathrm{~h}$ at $25^{\circ} \mathrm{C}$. YFP fluorescence signal was detected by laser scanning confocal microscopy. Arabidopsis protoplasts was prepared according to Axelos (1992).

\section{Gene Cloning and Plant Transformation}

Total RNA was isolated from the leaves of 14-day-old physic nut seedlings using TRIzol reagent (Invitrogen, Carlsbad, CA, USA) according to the manufacturer's instructions, and firststrand cDNA was obtained following Tang et al. (2016). JcDREB2 fragments including the complete coding sequences were amplified from physic nut by PCR with the primer pairs shown in Supplementary Table S1. The products were then cloned into the vector pMD 18-T (TaKaRa, Otsu, Japan) for use in sequencing and analysis. The target sequences were digested with the restriction enzymes Sac-I and Xba-I and then inserted

\footnotetext{
${ }^{1}$ http://www.ncbi.nlm.nih.gov/
} 
into the corresponding restriction sites of the pCAMBIA1301 vector under the control of a CaMV35S promoter. Next, Agrobacterium harboring the constructs was used to transform and regenerate rice seedlings, as described by Chen et al. (2003). In total we obtained seven independent transgenic lines, and selected three single gene insertion homozygous transgenic lines for further study based on an approximately 3:1 segregation ratio being observed among T2 plants. The segregation ratios, which were confirmed by GUS staining, were 216:70, 198:63 and 237:76. T2 seeds were germinated and 80 roots were taken from 10-day-old seedlings of each line of transgenic rice for GUS staining. GUS expression was observed in all roots from each line of 10-day-old seedlings.

\section{Stress Treatments}

After germination, rice seedlings were cultured in Yoshida's culture solution (Yoshida et al., 1976) at $25^{\circ} \mathrm{C}$ under $16 / 8 \mathrm{~h}$ (light/dark) conditions in a growth chamber. Two weeks later, seedlings were planted in soil in plastic pots and transferred to a greenhouse under natural sunlight. For $\mathrm{GA}_{3}$ treatment, wild-type and JcDREB2 overexpressing rice seeds were kept in water for 2 days for germination, then the seeds were incubated in Yoshida's culture solution containing $10 \mu \mathrm{M}$ $\mathrm{GA}_{3}$ for 10 days under natural sunlight. As an alternative GA treatment, plants grown in a greenhouse under natural sunlight were sprayed daily with $100 \mu \mathrm{M} \mathrm{GA}$ for 6 days (10-day-old seedlings) or 10 days (4-week-old seedlings). For the salt tolerance assay, 2-week-old seedlings were incubated in Yoshida's culture solution containing $150 \mathrm{mM} \mathrm{NaCl}$ for 5 days, followed by incubation in Yoshida's culture solution for 10 days at $25^{\circ} \mathrm{C}$ under $16 / 8 \mathrm{~h}$ (light/dark) conditions in a growth chamber, and then the survival rates were calculated. The relative electrolyte leakage (REL) and proline content were determined 2 days after salt treatment. Leaf samples were collected 2 days after salt treatment. All tests were repeated three times with three biological replicates for each test.

\section{Physiological Parameters, CAT and Superoxide Dismutase (SOD) Activity Measurements}

About $0.2 \mathrm{~g}$ of leaf sample was used for each determination. Firstly, each sample was washed five times with deionized water and placed in a test tube containing $10 \mathrm{~mL}$ of deionized water. The leaf samples were immersed and vibrated continuously at $25^{\circ} \mathrm{C}$ for $2 \mathrm{~h}$, then a conductivity meter was used to measure the electrical conductivity (B1) of the solution. Next the samples were boiled for $15 \mathrm{~min}$, and after cooling the solutions to room temperature, their conductivity (B2) was measured again. The REL was calculated according to the following formula: REL $(\%)=\mathrm{B} 1 / \mathrm{B} 2 \times 100$. The content of free proline in the rice leaves was determined as previously described (Bates et al., 1973). The content of malondialdehyde (MDA) in the leaves was determined as described by Cai et al. (2015). The activities of catalase (CAT) and superoxide dismutase (SOD) in the rice leaves were determined as previously described (Azooz et al., 2009).

\section{RNA Isolation and Expression Analysis}

Total RNA was isolated from the leaves of 14-day-old rice seedlings using TRIzol reagent (Invitrogen, USA) according to the manufacturer's instructions. Total RNA was extracted from roots, stem cortexes, flowers, and seeds of physic nut plants using a HiPure Plant RNA Mini kit (Promega, Madison, WI, USA) following the manufacturer's instructions. First strand cDNA was obtained by reverse transcription of total RNA using M-MLV reverse transcriptase (Promega, Madison, WI, USA) following the manufacturer's instructions. Primers used in the experiment are listed in Supplementary Table S1. Semi-quantitative RT-PCR with gene-specific primer pairs was used to detect the level of $J c D R E B 2$ gene expression in wild-type and transgenic plants. The OsUbiquitin gene was used as a control.

Quantitative real-time PCR was performed in a reaction volume of $20 \mu \mathrm{L}$ containing $2 \mu \mathrm{L}$ of cDNA, $10 \mu \mathrm{L}$ of $2 \times$ SYBR Premix ExTaq, $0.4 \mu \mathrm{L}$ forward primer $(10 \mu \mathrm{mol}), 0.4 \mu \mathrm{L}$ reverse primer $(10 \mu \mathrm{mol})$ and $7.2 \mu \mathrm{L}$ of ddH2O using a LCS480 system $\left(\right.$ Roch $\left.^{2}\right)$. Conditions for all quantitative real-time PCR amplifications were as follows: $95^{\circ} \mathrm{C}$ for $10 \mathrm{~min}$ for DNA polymerase activation, followed by 40 cycles of $95^{\circ} \mathrm{C}$ for $15 \mathrm{~s}$ and $60^{\circ} \mathrm{C}$ for $35 \mathrm{~s}$. The relative expression level was calculated using the $2^{-\Delta \Delta C T}$ method, and OsUbiquitin and JcActin were used as the reference genes for rice and physic nut, respectively. All experiments included three biological replicates, each with two technical replicates.

\section{Statistical Analysis}

All experiments included three biological replicates, and the data collected were analyzed using the Duncan multiple range test (Duncan, 1955) with the SAS software package ${ }^{3}$.

\section{RESULTS}

\section{Isolation and Phylogenetic Analysis of JcDREB2}

The full-length $J C D R E B 2$ cDNA was isolated from physic nut by reverse transcription PCR. The JCDREB2 sequence so obtained contained an open reading frame of 1074 bp that encoded a protein of 358 amino acids with one conserved AP2/ERF domain. The JCDREB2 sequence was deposited in the NCBI GenBank database under accession number JCGZ_24071. The conserved Val (14th) and Glu (19th) residues in the AP2/ERF domain are crucial in regulating the binding activity of DREB proteins to the DRE element (Cao et al., 2001). Further analysis of the deduced amino acid sequence indicated that the JcDREB2 protein contained these conserved 14th valine and 19th glutamic acid residues in the AP2/ERF domain and that it was highly homologous to DREB proteins from other plants (Figure 1A).

\footnotetext{
${ }^{2} \mathrm{http} / / /$ www.roche.com/

${ }^{3} \mathrm{http} / / /$ www.sas.com/en_us/software/sas9.html
} 
A

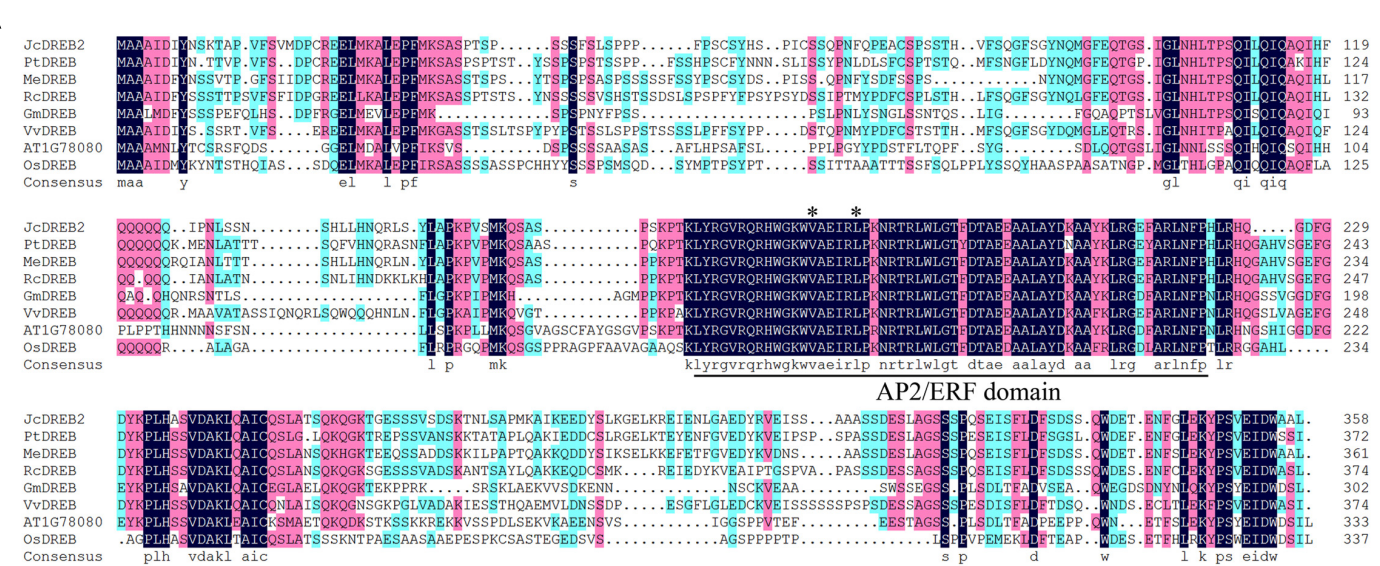

B

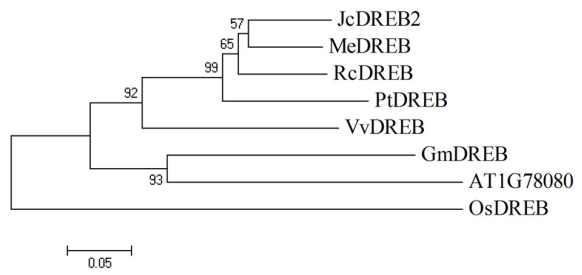

FIGURE 1 | Multiple sequence alignment and phylogenetic relationships of JcDREB2 homologs. (A) The conserved DREB AP2/ERF domain is indicated as the underlined segment. Stars indicate the amino acid residues in the AP2/ERF domain reported to be conserved. (B) Phylogenetic relationships of JcDREB2 homologs constructed using MEGA 5 software. The accession numbers of the protein sequences retrieved from GenBank and species designations are as follows: Me, Manihot esculenta (OAY35862); Pt, Populus trichocarpa (XP_002307098); Rc, Ricinus communis (XP_002533146); Gm, Glycine max (XP_003526587); Vv, Vitis vinifera (XP_003635449); Os, Oryza sativa (XP_015625871).

To further determine the relationship between JcDREB2 and other DREB proteins, a phylogenetic tree was established using the complete amino acid sequence (Figure 1B). Among the proteins included, the JcDREB2 protein was most closely related to cassava MeDREB, with a $75 \%$ match at the amino acid level (Figures 1A,B).

\section{Expression Patterns of JcDREB2}

To determine the expression pattern of JCDREB2 in physic nut, we analyzed its expression in five different tissues: roots, stem cortexes, leaves, flowers, and seeds by real-time quantitative PCR (qRT-PCR) using JcDREB2-specific primers. JcDREB2 was constitutively expressed in all of the tissues examined, but the highest level of expression was detected in leaves (Figure 2A).

Quantitative PCR analysis was performed to measure $J C D R E B 2$ expression under various abiotic stresses, including salt, $\mathrm{GA}_{3}$ and ABA. As shown in Figure 2B, JcDREB2 expression was repressed in physic nut leaves 1,3 , and $6 \mathrm{~h}$ after treatment with salt and $\mathrm{GA}_{3}$. However, under $\mathrm{ABA}$ stress, the level of JCDREB2 transcript was markedly up-regulated from 6 to $12 \mathrm{~h}$ after treatment.

\section{JcDREB2 Is a Nuclear-Localized Protein}

In order to determine the subcellular localization of the JcDREB2 protein, we examined the localization of JcDREB2-YFP by laser scanning confocal microscopy (Figure 3). The YFP fluorescence produced by 35 S::JCDREB2-YFP was localized in the nucleus, whereas YFP fluorescence produced by 35S::YFP was detected throughout the cell. These results indicate that JcDREB2 is a nuclear-localized protein.

\section{Overexpression of JcDREB2 in Rice Produces a Severe Dwarf Phenotype}

To investigate the function of $J C D R E B 2$, we overexpressed the $J C D R E B 2$ gene in rice under the control of the CaMV 35S promoter. Changes in levels of JCDREB2 transcripts in leaves were analyzed by semi-quantitative RT-PCR (Figure 4A). We observed that all transgenic rice plants overexpressing JcDREB2 (OeJcDREB2) had a severe dwarf phenotype under normal growing conditions (Figure 4B). The shoot and root length was shorter in OeJcDREB2 rice plants, compared with those of wild-type plants (Figures 4C,D).

\section{The Dwarf Phenotype of Transgenic Plants Is Rescued by the Application of Exogenous $\mathrm{GA}_{3}$}

Gibberellin, the most important of the hormones regulating shoot elongation, flowering, and seed development, plays an important role in determining plant height (Sakamoto et al., 2004). To examine whether the dwarf phenotype of OeJcDREB2 rice plants was caused by GA deficiency, we cultivated wild-type and transgenic seedlings in Yoshida's culture solution containing $10 \mu \mathrm{M} \mathrm{GA}{ }_{3}$ for 10 days at the germination stage. Under these 

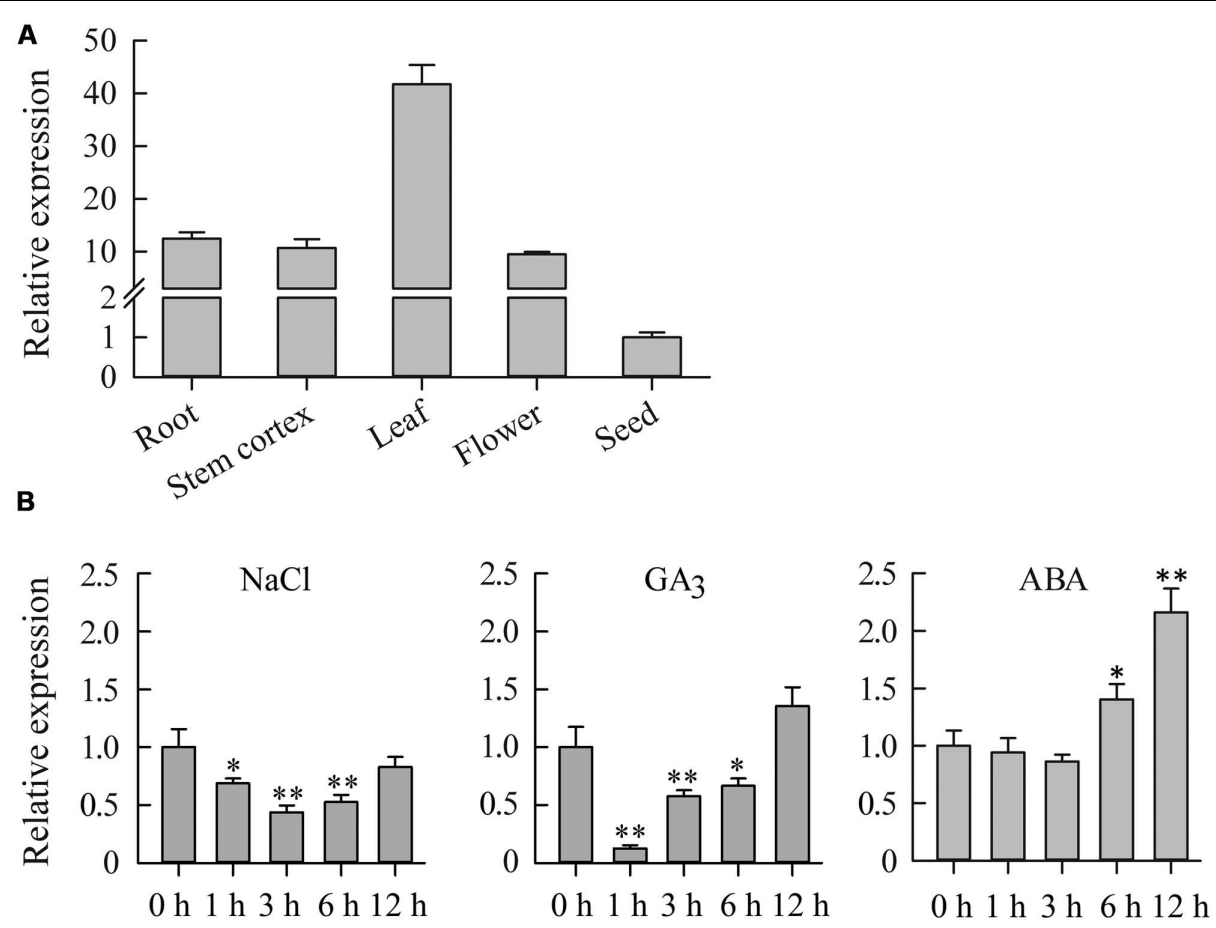

FIGURE 2 | Expression analysis of the JcDREB2 gene in physic nut. (A) qRT-PCR analysis of JCDREB2 gene expression in roots, stem cortex, leaves, flowers, and seeds at 35 days after pollination. Relative expression was normalized with respect to the reference gene JcActin (internal control). Bars show means $\pm \mathrm{SD}$ of three biological replicates. (B) qRT-PCR analysis of JCDREB2 gene expression in leaves under different abiotic stresses. Bars show standard deviations of the replicates. Each assay was run in triplicate for three independent biological replicates. Values represent means of $n=3 \pm \mathrm{SD}\left(\mathrm{Duncan}\right.$ test: $\left.{ }^{*} P<0.05,{ }^{*} P<0.01\right)$.
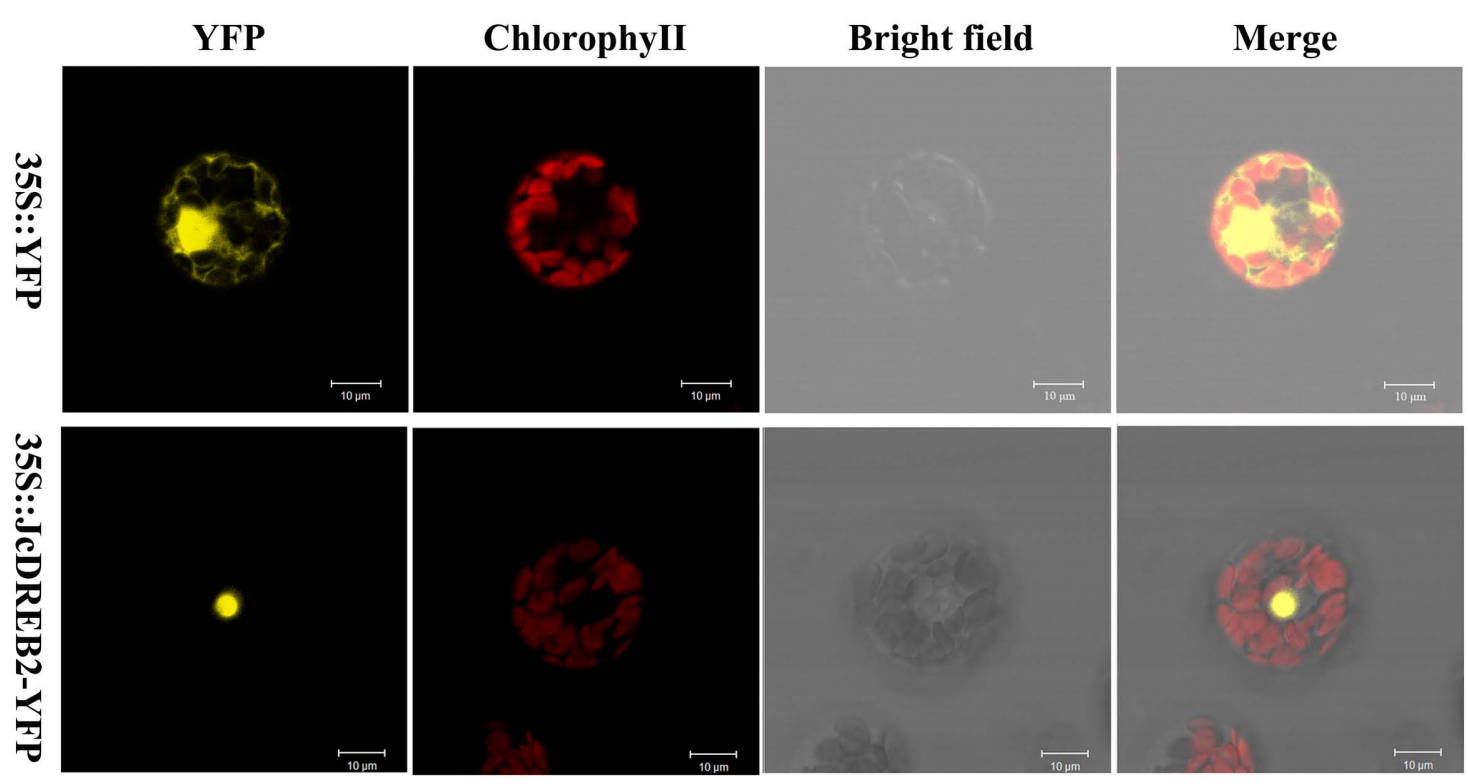

FIGURE 3 | Subcellular localization of JcDREB2.

conditions, shoots of transgenic as well as of wild-type plants showed rapid elongation (Figures 5A,D), but the elongation ratio $\left(+\mathrm{GA}_{3} /-\mathrm{GA}_{3}\right)$ of OeJcDREB2 plants was significantly higher than that of the wild-type (Figure 5G). Furthermore, spraying OeJcDREB2 rice plants with $100 \mu \mathrm{M} \mathrm{GA}_{3}$ rescued shoot elongation; in particular, the length of the leaf sheath was fully restored to a level similar to that in wild-type plants (Figures 5B,C,E,F). The elongation ratio $\left(+\mathrm{GA}_{3} /-\mathrm{GA}_{3}\right)$ of 

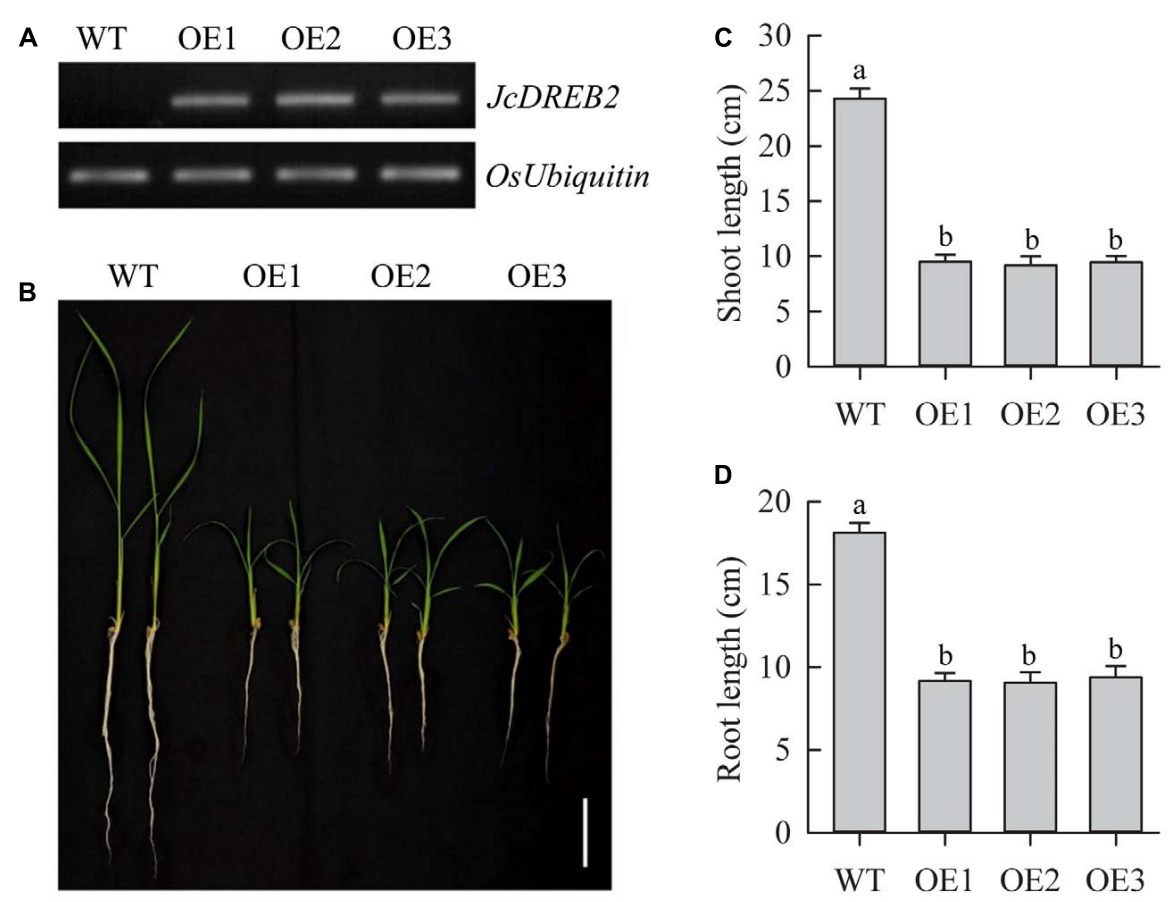

FIGURE 4 | Phenotype of JcDREB2-overexpressing rice plants. (A) Relative levels of JCDREB2 transcript in different transgenic rice plants (OE1, OE2, and OE3), determined by semi-quantitative RT-PCR; (B) Phenotype of wild-type and dwarf JCDREB2-overexpressing plants. Two-week-old seedlings cultured in water were photographed. Bar $=5 \mathrm{~cm}$. (C,D) Relative shoot length $\mathbf{( C )}$ and root length (D) in wild-type plants and three JcDREB2-overexpressing lines after 14 days of growth on Yoshida's culture solution. The experiment included three biological replicates. Values represent (C,D) means of $n=20 \pm \mathrm{SD}$ from three independent experiments and different letters above the columns indicate significant differences from the corresponding WT at the $p<0.01$ level.

OeJcDREB2 plants was also significantly higher than that of the wild-type (Figures $\mathbf{5 H}, \mathbf{I}$ ). These results suggest that OeJcDREB2 rice plants do indeed exhibit a classic reduced-GA phenotype.

\section{GA Biosynthesis Is Regulated by JcDREB2 Expression}

To investigate whether overexpression of JcDREB2 altered the expression of GA biosynthesis genes, we used qRT-PCR assays to detect the expression of genes encoding two key enzymes [gibberellin 20-oxidase (GA20ox) and gibberellin 3 $\beta$-hydroxylase (GA3ox)] as well as other genes responsible for the early steps in GA biosynthesis, including OsCPS1, OsKO2, and OsKAO. As shown in Figure 6, the expression levels of OsGA20ox1, OsGA20ox2, OsGA20ox4, OsGA3ox2, OsCPS1, OsKO2, and OsKAO exhibited a significant reduction in OeJcDREB2 seedlings compared with their levels in wild-type. GID2 (gibberellininsensitive dwarf2) is involved in the GA signaling pathway in rice (Gomi et al., 2004). We therefore also examined the expression levels of the GID2 gene, but no significant difference in GID2 expression was seen between OeJcDREB2 and wild-type plants (Figure 6).

\section{JcDREB2 Negatively Regulates the Salinity-Induced Response in Rice}

To examine whether $J C D R E B 2$ plays a role in salt stress tolerance, we compared the salt tolerance of OeJcDREB2 and wild-type plants at the vegetative growth stage. After 5 days of salt treatment with $150 \mathrm{mM} \mathrm{NaCl}, 73.3 \%$ of wild-type seedlings remained green, whereas OeJcDREB2 seedlings showed severe leaf aging and rolling (Figure $7 \mathbf{A}$ ). The survival rates of wild-type and $O e J c D R E B 2$ seedlings after 10 days of recovery were statistically analyzed. Our data showed that approximately $38 \%$ of the wild-type seedlings survived, whereas no OeJcDREB2 seedlings survived after the recovery period (Figure 7B). However, overexpressing JcDREB2 had no significant effect on drought tolerance (data not shown) in rice. Proline accumulation is considered to be a plant adaptive response to high salinity and drought stresses (Xiong et al., 2012). Under normal growth conditions, the proline content did not differ between wildtype and OeJcDREB2 plants. However, under salt treatment, the proline content of OeJcDREB2 plants was significantly lower than that of the wild-type (Figure $7 \mathrm{C}$ ). This finding indicated that salt stress was more damaging to the OeJcDREB2 plants than to the wild-type plants. Electrolyte leakage is an important index of cell membrane damage in plant stress responses. The relative level of electrolyte leakage from wild-type leaves was lower than that from OeJcDREB2 leaves after the salt stress treatments (Figure 7D).

Membranes abound in polyunsaturated fatty acids which serve as antioxidants by capturing reactive oxygen species, thereby protecting plants from being damaged under extreme conditions. This process generates MDA, a typical marker of the oxidation of polyunsaturated fatty acids. The MDA 
A

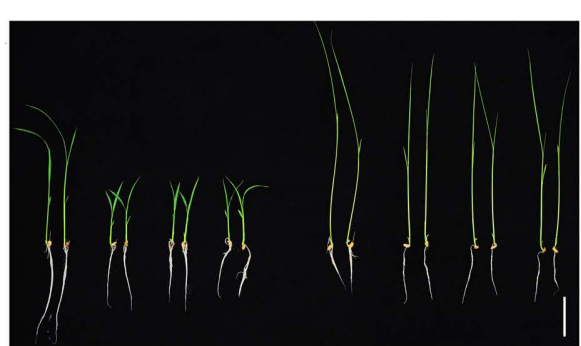

B

c
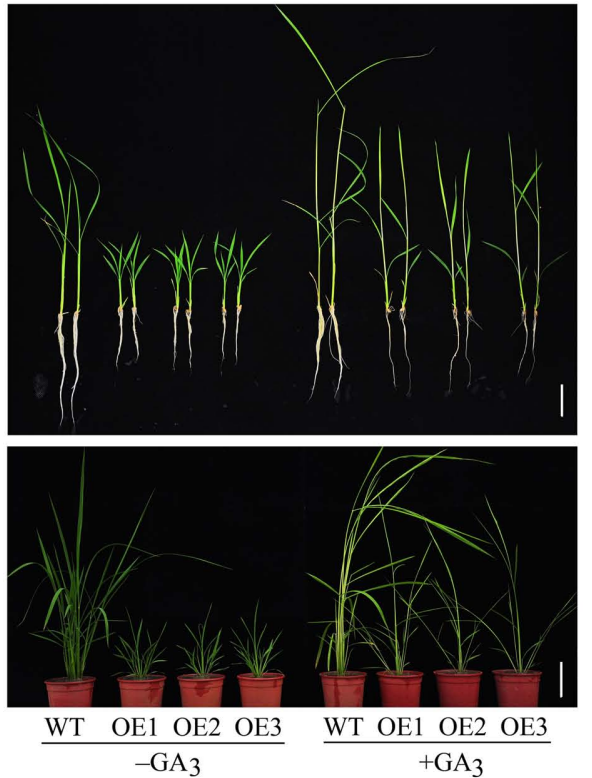

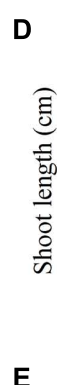
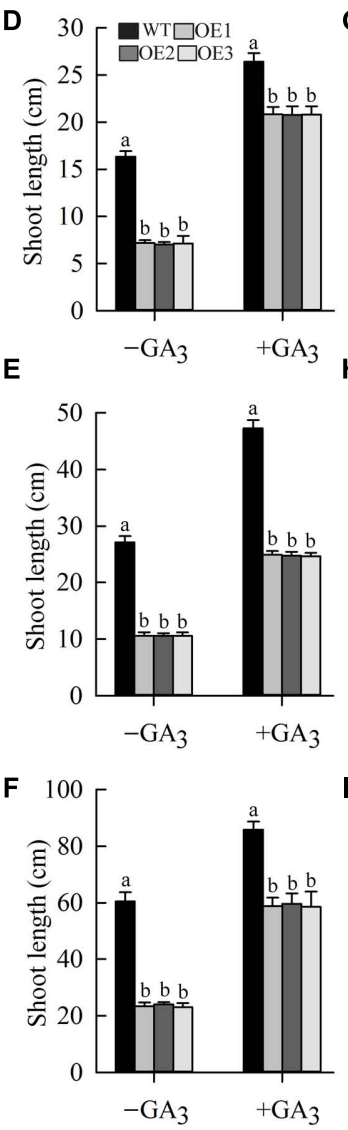

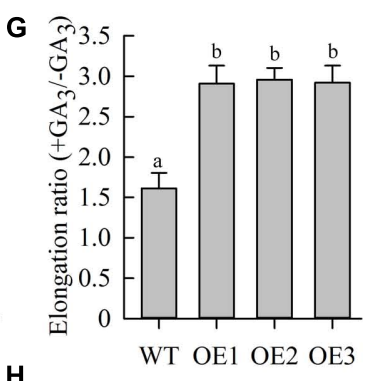

H
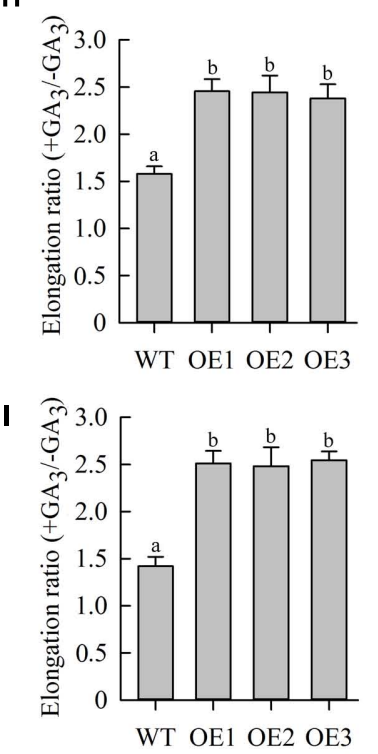

FIGURE 5 | Exogenous $\mathbf{G A}_{3}$ effectively reverses the GA-deficiency phenotype. (A) Ten-day-old seedlings germinated and grown in Yoshida's culture solution containing $10 \mu \mathrm{M} \mathrm{GA}$ or no $\mathrm{GA}_{3}$. Bar $=5 \mathrm{~cm}$; (B) $\mathrm{GA}_{3}$ treatment. Ten-day-old seedlings were sprayed with $100 \mu \mathrm{M} \mathrm{GA} \mathrm{A}_{3}$ daily for 6 days. Bar $=5 \mathrm{~cm}$. (C) $\mathrm{GA} 3$ treatment. Four-week-old seedlings were sprayed with $100 \mu \mathrm{M} \mathrm{GA} 3$ daily for 10 days. Bar $=10 \mathrm{~cm}$. (D) Relative shoot length was measured on wild-type plants and

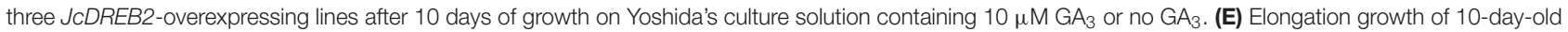

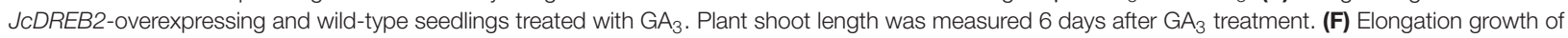
4-week-old $J_{C D R E B 2}$-overexpressing and wild-type seedlings treated with $\mathrm{GA}_{3}$. Plant shoot length was measured 10 days after $\mathrm{GA} \mathrm{A}_{3}$ treatment. (G) Elongation ratio $\left(+\mathrm{GA}_{3} /-\mathrm{GA}_{3}\right)$ of 10 -day-old seedlings germinated and grown in Yoshida's culture solution. $(\mathbf{H})$ Elongation ratio ( $\left.+\mathrm{GA} / \mathrm{A}_{3}-\mathrm{GA}\right)$ of 10 -day-old seedlings grown with $\mathrm{GA}_{3}$ or without $\mathrm{GA}_{3}$ for 6 days. (I) Elongation ratio ( $+\mathrm{GA}_{3} /-\mathrm{GA}_{3}$ ) of 4-week-old $J c D R E B 2$-overexpressing and wild-type seedlings grown with $\mathrm{GA} \mathrm{A}_{3}$ or without $\mathrm{GA} \mathrm{A}_{3}$ for 10 days. The experiment included three biological replicates. Values represent (D-I) means of $n=15 \pm$ SD from three independent experiments and different letters above the columns indicate significant differences from the corresponding WT at the $p<0.01$ level.

content of wild-type plants was lower than that of OeJcDREB2 plants under salt treatment (Figure 7E). These results suggested that there was more cell membrane damage in OeJcDREB2 leaf cells than in wild-type leaf cells under salt stress.

Superoxide dismutase is an antioxidant enzyme that catalyzes the conversion of the superoxide radical into hydrogen peroxide, and CAT further catalyzes the transformation of harmful hydrogen peroxide into harmless water, so protecting plants from damage by reactive oxygen species arising as a result of abiotic stress. We analyzed CAT and SOD activities under salinity stress and normal conditions. The results suggested that the activities of CAT and SOD from OeJcDREB2 leaves were lower than those from wild-type leaves under salinity stress, whereas no significant difference was found under normal growth conditions (Figures 7F,G).
Finally, we measured the expression levels of several genes which have previously been shown to respond to salt stress in rice seedlings, since overexpressing these genes could improve the salinity stress tolerance of transgenic plants. The products of the OsHKT1;1 gene are crucial for salt tolerance in rice through the exclusion of $\mathrm{Na}^{+}$ions from sensitive cells (Ren et al., 2005). The OsGR3 gene products are involved in scavenging reactive oxygen species (Wu et al., 2015). Increases in the levels of expression of OsHKT1;1, OsP5CS and OsGR3 under salt treatment were strongly impaired in the leaves of two OeJcDREB2 plants (Figure $\mathbf{7 H}$ ). In addition, the expression level of the salt-stress-related transcription factor gene SNAC1 (STRESSRESPONSIVE NAC 1) (Hu et al., 2006) was significantly lower in $O e J c D R E B 2$ leaves than in wild-type leaves under salinity stress (Figure 7H). 

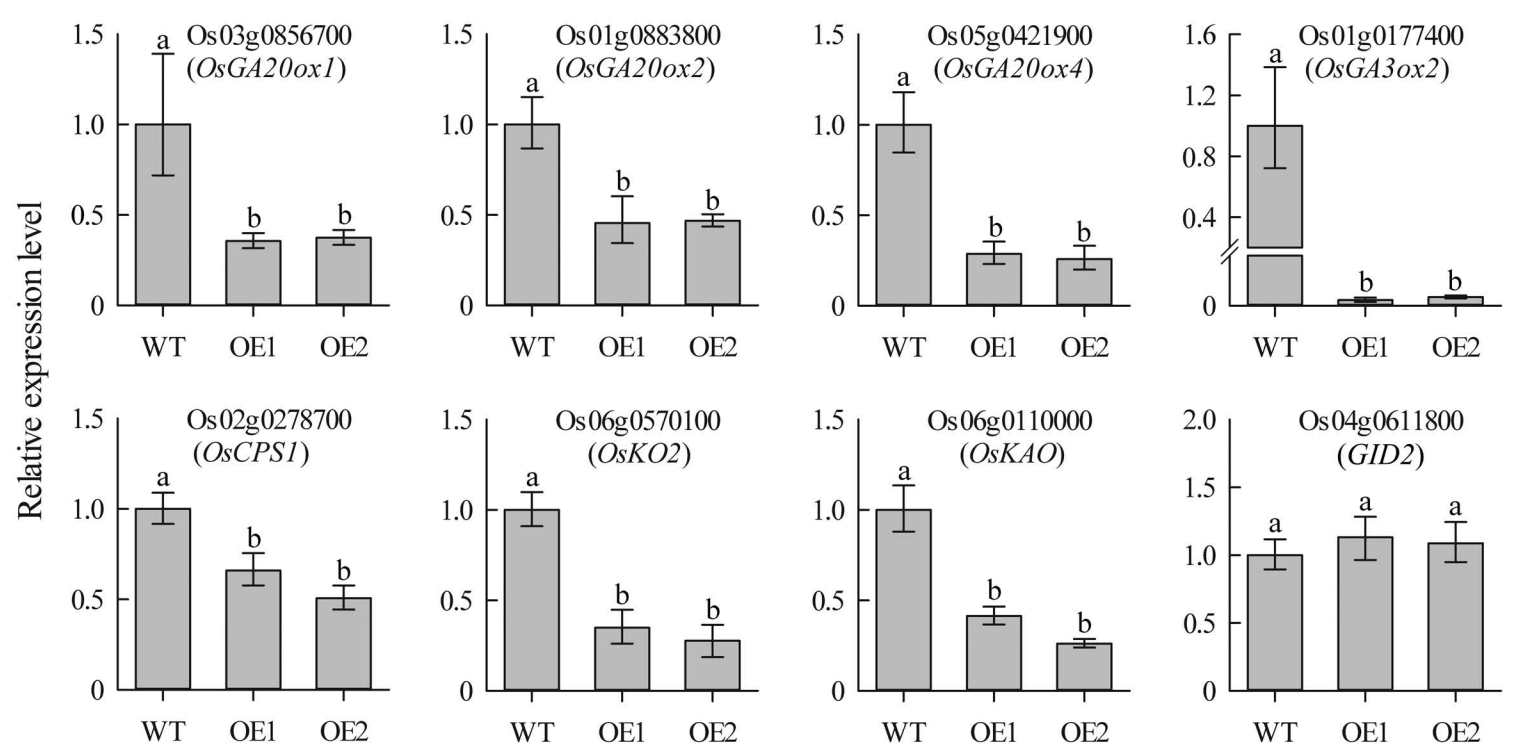

FIGURE 6 | Real-time PCR analysis of GA biosynthesis genes in leaves of 2-week-old seedlings. Each PCR assay was run in duplicate for each of three independent biological replicates. Values represent means of $n=6 \pm \mathrm{SD}$ and different letters above the columns indicate significant differences from the corresponding WT at the $p<0.01$ level.

\section{DISCUSSION}

The AP2/ERF proteins constitute quite a large family, with 119 members, in physic nut (Tang et al., 2016). Although at least 5 physic nut AP2/ERF genes, JcERF (Tang et al., 2007), JcDREB (Tang et al., 2011), JcERF1 (Yang et al., 2014), JcERF2 (Wang et al., 2015), and JcERF011 (Tang et al., 2016), have been shown to play significant roles in plant development and abiotic stress responses, the biological function of most JcAP2/ERF genes is not known. In this study, we show that JCDREB2 is a stress-responsive AP2/ERF gene and that, when overexpressed in rice, it has significant effects on plant growth and salinity stress.

Our results demonstrated that overexpression of JcDREB2 in rice produced plants that were dwarfed with retarded growth (Figure 4B). The dwarf phenotype of OeJcDREB2 plants could be restored by the application of $\mathrm{GA}_{3}$ (Figure 5). This shows that the dwarf phenotype is the result of a deficiency of bioactive GAs in the transgenic rice. These results are consistent with previous studies on GA-deficiency mutants in a wide range of plants, including barley (Jia et al., 2009), Arabidopsis (Barboza et al., 2013), potato (Roumeliotis et al., 2013), maize (Chen et al., 2014), and rice (Oikawa et al., 2004; Qin et al., 2013).

We also found that overexpression of JcDREB2 in rice restricted shoot growth by inhibiting the expression of OsGA20ox1, OsGA20ox2, OsGA20ox4, OsGA3ox2, OsCPS1, OsKO2, and OsKAO, which encode key enzymes in the GA biosynthetic pathway. There is accumulating evidence to indicate that targeting GA20ox, GA3ox, CPS, KO2 and KAO genes results in decreased levels of bioactive GA and dwarf plants. GA20ox and GA3ox catalyze the last two steps in active GA biosynthesis, converting inactive forms of GA such as GA20 into active forms such as GA1 (Roumeliotis et al., 2013). Downregulating the expression of GA20ox and GA3ox causes severe dwarf phenotypes by altering levels of active GAs (Carrera et al., 2000; Itoh et al., 2004; Roumeliotis et al., 2013; Regnault et al., 2014). Similarly, the CPS gene, which encodes ent-copalyl diphosphate synthase, and mutated forms of the gene, have been isolated from various plants, and a typical phenotype in these mutants is dwarfism (Sakamoto et al., 2004). Ent-kaurenoic acid oxidase (KAO) catalyzes the conversion of ent-kaurenoic acid (KA) to gibberellin GA12, the precursor of all GAs (Regnault et al., 2014). The kao1 kao2 double mutant exhibits a typical GA-deficient dwarf phenotype (Regnault et al., 2014). Taken together, our results suggest that $J C D R E B 2$ restricts shoot growth by downregulating the expression of key GA biosynthetic enzymes. GID2 is a positive regulator of GA signaling which is essential for the GA-mediated degradation of DELLA proteins such as SLR1 (slender Rice 1) (Gomi et al., 2004). Our study suggests that the expression of GID2 is not significantly changed in JCDREB2 overexpressers (Figure 6). This result further demonstrates that JCDREB2 negatively regulates shoot growth processes by repressing GA biosynthesis and not via signal transduction.

A large number of studies have reported that many DREB gene products are involved in abiotic stress responses and that overexpression of $D R E B$ genes can enhance plant tolerance of abiotic stress (Lata and Prasad, 2011). In the present study, we found that the expression of JcDREB2 in physic nut leaves was repressed by salinity stress (Figure 2B). Overexpressing the $J c D R E B 2$ gene in rice reduced its tolerance to salt stress, leading to more pronounced leaf aging and rolling (Figure 7A). When plants suffer from drought and salinity stresses, there are rapid physiological changes in response to these adverse conditions 

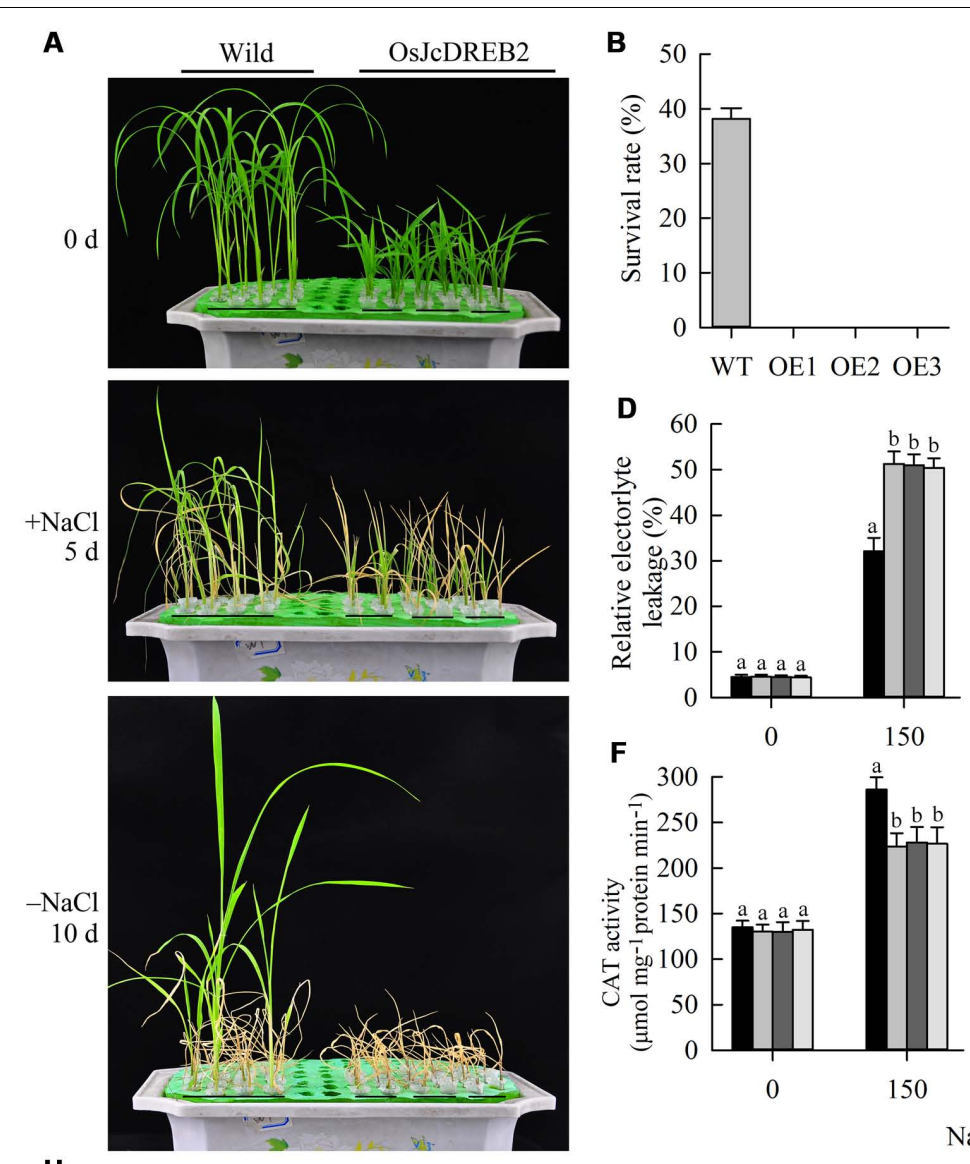

H
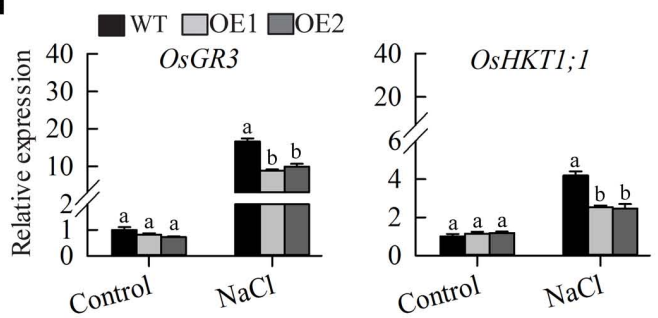

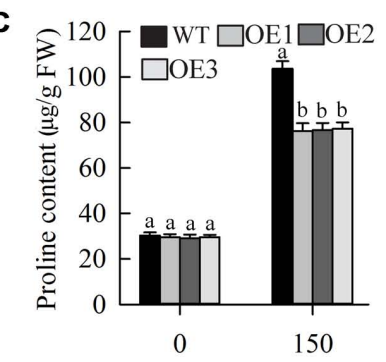

E
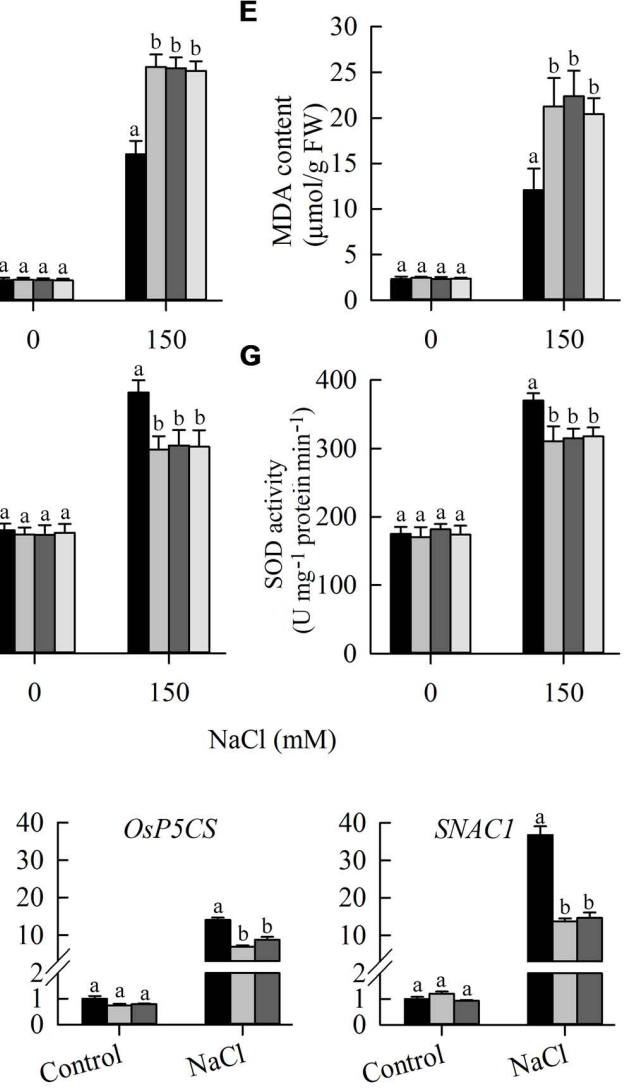

FIGURE 7 | Salt stress tolerance tests on JcDREB2-overexpressing rice lines and wild-type plants. (A) Performance of wild-type and transgenic plants before and after salt treatment (150 mM NaCl), and after recovery for 10 days following salt treatment. The experiment included three biological replicates.

(B) Survival rates of wild-type and transgenic plants after recovery for 10 days following salt treatment. (C) Proline content of leaves before and after salt treatment. (D,E) Relative electrolyte leakage (REL) (D) and MDA content (E) of leaves before and after salt treatment. Values represent means of $n=15 \pm$ SD from three independent experiments and different letters above the columns indicate significant differences from the corresponding WT at the $p<0.01$ level. (F,G) Activity of catalase (CAT) (F) and superoxide dismutase (SOD) (G) in leaves before and after salt treatment. Values represent means of $n=15 \pm$ SD from three independent experiments and different letters above the columns indicate significant differences from the corresponding WT at the $p<0.05$ level. (H) Relative expression levels of salt stress-responsive genes. The experiment included three biological replicates, each with two technical replicates. Values represent means of $n=6 \pm \mathrm{SD}$ and different letters above the columns indicate significant differences from the corresponding WT at the $p<0.01$ level.

(Seki et al., 2007). Consequently, physiological signs related to the plants' responses to abiotic stress can be used as reference points in the evaluation of plant stress tolerance. Proline is considered to play an important part in defense mechanisms (Nanjo et al., 1999). A strong link between tolerance of abiotic stress and the accumulation of proline has been demonstrated by knocking out and overexpressing the P5CS gene in a number of plants (Nanjo et al., 1999; Bartels and Sunkar, 2007). Our study suggested that there was less accumulation of proline in the transgenic rice plants compared with the wild-type rice plants under salinity stress (Figure 7C), indicating that proline may be one factor responsible for the low tolerance shown by JcDREB2 transgenic plants toward salinity stress. Furthermore, the leaves of the transgenic rice plants showed higher REL and MDA content compared with the leaves of wild-type rice (Figures 7D,E), showing that the wild-type plants were better able to resist the adverse effects of salinity stress. SOD and CAT play important roles in protecting plants against the toxic effects of reactive 
oxygen species produced as a result of various abiotic stresses (Azooz et al., 2009). Our study indicated that there was higher SOD and CAT activity in the wild-type rice plants compared with the transgenic rice plants under salinity stress (Figures 7F,G), which suggests that the transgenic rice plants were disadvantaged under conditions of high salinity. Since transcriptional regulatory networks responsive to salinity and drought stresses have been identified in rice and Arabidopsis, research into the expression of salinity stress-responsive genes in the networks could supply information crucial for dissecting the functions of $J C D R E B 2$ in salinity stress tolerance. Our results indicated that the expression of stress-responsive genes such as OsHKT1;1, OsP5CS, OsGR3, and SNAC1 was significantly lower in transgenic rice compared to wild-type under salinity stress (Figure $7 \mathbf{H}$ ). Related research has shown that overexpressing these genes may improve the tolerance of transgenic plants to salinity stress (Ren et al., 2005; Hu et al., 2006; Wu et al., 2015). Our findings indicate that JcDREB2 affected salt stress tolerance in the transgenic rice plants at least partially owing to decreases in the expression of stress-responsive genes under salinity stress.

\section{CONCLUSION}

We characterized the physic nut AP2/ERF gene JcDREB2, the expression of which was repressed by salinity and $\mathrm{GA}_{3}$, but was induced by ABA. Overexpression of JcDREB2 in rice resulted in dwarf and GA-deficient phenotypes, and these phenotypes were restored by exogenous $\mathrm{GA}_{3}$ treatment. Overexpression of JCDREB2 enhanced sensitivity to salt stress in transgenic rice

\section{REFERENCES}

Axelos, M. (1992). A protocol for transient gene expression in Arabidopsis thaliana protoplasts isolated from cell suspension cultures. Plant Physiol. Biochem. 30, $123-128$.

Azooz, M. M., Ismail, A. M., and Elhamd, M. F. A. (2009). Growth, lipid peroxidation and antioxidant enzyme activities as a selection criterion for the salt tolerance of maize cultivars grown under salinity stress. Int. J. Agric. Biol. $11,572-577$.

Barboza, L., Effgen, S., Alonsoblanco, C., Kooke, R., Keurentjes, J. J., and Koornneef, M. (2013). Arabidopsis semidwarfs evolved from independent mutations in GA20ox1, ortholog to green revolution dwarf alleles in rice and barley. Proc. Natl. Acad. Sci. U.S.A. 110, 15818-15823. doi: 10.1073/pnas. 1314979110

Bartels, D., and Sunkar, R. (2007). Drought and salt tolerance in plants. Crit. Rev. Plant Sci. 24, 23-58. doi: 10.1080/07352680590910410

Bates, L. S., Waldren, R. P., and Teare, I. D. (1973). Rapid determination of free proline for water-stress studies. Plant Soil 39, 205-207. doi: 10.1007/ BF00018060

Cai, W., Liu, W., Wang, W. S., Fu, Z. W., Han, T. T., and Lu, Y. T. (2015). Overexpression of rat neurons nitric oxide synthase in rice enhances drought and salt tolerance. PLoS ONE 10:e0131599. doi: 10.1371/journal.pone.0131599

Cao, Z. F., Li, J., Chen, F., Li, Y. Q., Zhou, H. M., and Liu, Q. (2001). Effect of two conserved amino acid residues on DREB1A function. Biochemistry (Moscow) 66, 623-627. doi: 10.1023/A:1010251129429

Carrera, E., Bou, J., Garcíamartínez, J. L., and Prat, S. (2000). Changes in GA 20-oxidase gene expression strongly affect stem length, tuber induction and plants. These results increase our understanding of the roles played by this physic nut AP2/ERF transcription factor in plant growth and responses to abiotic stress.

\section{AUTHOR CONTRIBUTIONS}

The research was conceived and designed by YT and CL. The experiments were performed by YT, and the data were analyzed by YT, KL, JZ, XL, KX, YZ, JQ, DY, and JW. The manuscript was written and revised by YT and CL. All the authors read and approved the final manuscript.

\section{ACKNOWLEDGMENTS}

This research was supported by grants from the National Natural Science Foundation of China (Nos. 31272168, 31071807), Science and Technology Open Cooperation Project of Henan Province (No. 132106000077), Foundation of He'nan Educational Committee (No. 17B180008 and No. 16A180057), Foundation of He'nan Science and Technology Committee (No. 152300410229, No. 142102210108, No. 152102410074, and No. 144300510072), and the Startup Fund for Advanced Talents of Zhoukou Normal University (No. ZKNUC2016030).

\section{SUPPLEMENTARY MATERIAL}

The Supplementary Material for this article can be found online at: http://journal.frontiersin.org/article/10.3389/fpls.2017.00306/ full\#supplementary-material

tuber yield of potato plants. Plant J. 22, 247-256. doi: 10.1046/j.1365-313x.2000. 00736.x

Chen, H., Liu, L., Wang, L., Wang, S., and Cheng, X. (2015). VrDREB2A, a DREB-binding transcription factor from Vigna radiata, increased drought and high-salt tolerance in transgenic Arabidopsis thaliana. J. Plant Res. 129, 1-11. doi: 10.1007/s10265-015-0773-0

Chen, M., Zhao, Y., Zhuo, C., Lu, S., and Guo, Z. (2015). Overexpression of a NFYC transcription factor from bermudagrass confers tolerance to drought and salinity in transgenic rice. Plant Biotechnol. J. 13, 482-491. doi: 10.1111/pbi. 12270

Chen, S., Jin, W., Wang, M., Fan, Z., Jie, Z., Jia, Q., et al. (2003). Distribution and characterization of over 1000 T-DNA tags in rice genome. Plant J. 36, 105-113. doi: 10.1046/j.1365-313X.2003.01860.x

Chen, Y., Hou, M., Liu, L., Wu, S., Shen, Y., Ishiyama, K., et al. (2014). The maize DWARF1 encodes a gibberellin 3-oxidase and is dual localized to the nucleus and cytosol. Plant Physiol. 166, 2028-2039. doi: 10.1104/pp.114. 247486

Duncan, D. B. (1955). Multiple range and multiple F tests. Biometrics 11, 1-42. Gomi, K., Sasaki, A., Itoh, H., Ueguchi-Tanaka, M., Ashikari, M., Kitano, H., et al. (2004). GID2, an F-box subunit of the SCF E3 complex, specifically interacts with phosphorylated SLR1 protein and regulates the gibberellin-dependent degradation of SLR1 in rice. Plant J. 37, 626-634. doi: 10.1111/j.1365-313X. 2003.01990.x

Hong, Y., Zhang, H., Huang, L., Li, D., and Song, F. (2016). Overexpression of a stress-responsive NAC transcription factor gene ONAC022 improves drought and salt tolerance in rice. Front. Plant Sci. 7:4. doi: 10.3389/fpls.2016. 00004 
Hu, H., Dai, M., Yao, J., Xiao, B., Li, X., Zhang, Q., et al. (2006). Overexpressing a NAM, ATAF, and CUC (NAC) transcription factor enhances drought resistance and salt tolerance in rice. Proc. Natl. Acad. Sci. U.S.A. 103, 12987-12992. doi: 10.1073/pnas.0604882103

Itoh, H., Tatsumi, T., Sakamoto, T., Otomo, K., Toyomasu, T., Kitano, H., et al. (2004). A rice semi-dwarf gene, tan-ginbozu ( D35 ), Encodes the gibberellin biosynthesis enzyme, ent -kaurene oxidase. Plant Mol. Biol. 54, 533-547. doi: 10.1023/B:PLAN.0000038261.21060.47

Jia, Q., Zhang, J., Westcott, S., Zhang, X. Q., Bellgard, M., Lance, R., et al. (2009). GA-20 oxidase as a candidate for the semidwarf gene sdw1/ denso in barley. Funct. Integr. Genomics 9, 255-262. doi: 10.1007/s10142-0090120-4

Jiang, Q., Zheng, H., Zhang, H., and Ma, Y. (2014). Overexpression of GmDREB1 improves salt tolerance in transgenic wheat and leaf protein response to high salinity. Crop J. 2, 120-131. doi: 10.1016/j.cj.2014.02.003

Jin, T., Chang, Q., Li, W., Yin, D., Li, Z., Wang, D., et al. (2010). Stressinducible expression of GmDREB1 conferred salt tolerance in transgenic alfalfa. Plant Cell Tissue Organ Cult. 100, 219-227. doi: 10.1007/s11240-0099628-5

Lata, C., and Prasad, M. (2011). Role of DREBs in regulation of abiotic stress responses in plants. J. Exp. Bot. 62, 4731-4748. doi: 10.1093/jxb/ err210

Li, F., Guo, S., Zhao, Y., Chen, D., Chong, K., and Xu, Y. (2010). Overexpression of a homopeptide repeat-containing bHLH protein gene (OrbHLH001) from dongxiang wild rice confers freezing and salt tolerance in transgenic Arabidopsis. Plant Cell Rep. 29, 977-986. doi: 10.1007/s00299-0100883-z

Nanjo, T., Kobayashi, M., Yoshiba, Y., Sanada, Y., Wada, K., Tsukaya, H., et al. (1999). Biological functions of proline in morphogenesis and osmotolerance revealed in antisense transgenic Arabidopsis thaliana. Plant J. 18, 185-193. doi: 10.1046/j.1365-313X.1999.00438.x

Oikawa, T., Koshioka, M., Kojima, K., Yoshida, H., and Kawata, M. (2004). A role of OsGA20ox1, encoding an isoform of gibberellin 20-oxidase, for regulation of plant stature in rice. Plant Mol. Biol. 55, 687-700. doi: 10.1007/s11103-004$1692-y$

Openshaw, K. (2000). A review of Jatropha curcas: an oil plant of unfulfilled promise. Biomass Bioenergy 19, 1-15. doi: 10.1016/S0961-9534(00) 00019-2

Qin, X., Liu, J. H., Zhao, W. S., Chen, X. J., Guo, Z. J., and Peng, Y. L. (2013). Gibberellin 20-oxidase gene OsGA20ox3 regulates plant stature and disease development in rice. Mol. Plant Microbe Interact. 26, 227-239. doi: 10.1094/ MPMI-05-12-0138-R

Regnault, T., Davière, J. M., Heintz, D., Lange, T., and Achard, P. (2014). The gibberellin biosynthetic genes AtKAO1 and AtKAO2 have overlapping roles throughout Arabidopsis development. Plant J. 80, 462-474. doi: 10.1111/tpj. 12648

Ren, Z. H., Gao, J. P., Li, L. G., Cai, X. L., Huang, W., Chao, D. Y., et al. (2005). A rice quantitative trait locus for salt tolerance encodes a sodium transprter. Nat. Genet. 37, 1141-1146. doi: 10.1038/ng1643

Roumeliotis, E., Kloosterman, B., Oortwijn, M., Lange, T., Visser, R. G. F., and Bachem, C. W. B. (2013). Down regulation of StGA3ox genes in potato results in altered GA content and affect plant and tuber growth characteristics. J. Plant Physiol. 170, 1228-1234. doi: 10.1016/j.jplph.2013.04.003

Sakamoto, T., Miura, K., Itoh, H., Tatsumi, T., Ueguchitanaka, M., Ishiyama, K., et al. (2004). An overview of gibberellin metabolism enzyme genes and their related mutants in rice. Plant Physiol. 134, 1642-1653. doi: 10.1104/pp.103. 033696

Sakuma, Y., Liu, Q., Dubouzet, J. G., Abe, H., Shinozaki, K., and YamaguchiShinozaki, K. (2002). DNA-Binding specificity of the ERF/AP2 domain of Arabidopsis DREBs, transcription factors involved in dehydration- and coldinducible gene expression. Biochem. Biophys. Res. Commun. 290, 998-1009. doi: 10.1006/bbrc.2001.6299

Seki, M., Umezawa, T., Urano, K., and Shinozaki, K. (2007). Regulatory metabolic networks in drought stress responses. Curr. Opin. Plant Biol. 10, 296-302. doi: 10.1016/j.pbi.2007.04.014

Shao, H., Wang, H., and Tang, X. (2015). NAC transcription factors in plant multiple abiotic stress responses: progress and prospects. Front. Plant Sci. 6:902. doi: $10.3389 /$ fpls.2015.00902
Shinozaki, K., and Yamaguchi-Shinozaki, K. (2000). Molecular responses to dehydration and low temperature: differences and cross-talk between two stress signaling pathways. Curr. Opin. Plant Biol. 3, 217-223. doi: 10.1016/S13695266(00)80068-0

Singh, K. B., Foley, R. C., and Oñate-Sánchez, L. (2002). Transcription factors in plant defense and stress responses. Curr. Opin. Plant Biol. 5, 430-436. doi: 10.1016/S1369-5266(02)00289-3

Song, C., Je, J., Hong, J. K., and Lim, C. O. (2014). Ectopic expression of an Arabidopsis dehydration-responsive element-binding factor DREB2C improves salt stress tolerance in crucifers. Plant Cell Rep. 33, 1239-1254. doi: 10.1007/ s00299-014-1612-9

Sun, S., Yu, J. P., Chen, F., Zhao, T. J., Fang, X. H., Li, Y. Q., et al. (2008). TINY, a dehydration-responsive element (DRE)-binding protein-like transcription factor connecting the DRE- and ethylene-responsive elementmediated signaling pathways in Arabidopsis. J. Biol. Chem. 283, 6261-6271. doi: 10.1074/jbc.M706800200

Tamura, K., Peterson, D., Peterson, N., Stecher, G., Nei, M., and Kumar, S. (2011). MEGA5 : molecular evolutionary genetics analysis using maximum likelihood, evolutionary distance, and maximum parsimony methods. Mol. Biol. Evol. 28, 2731-2739. doi: 10.1093/molbev/msr121

Tang, M., Liu, X., Deng, H., and Shen, S. (2011). Over-expression of JcDREB, a putative AP2/EREBP domain-containing transcription factor gene in woody biodiesel plant Jatropha curcas, enhances salt and freezing tolerance in transgenic Arabidopsis thaliana. Plant Sci. 181, 623-631. doi: 10.1016/j.plantsci. 2011.06.014

Tang, M., Sun, J., Liu, Y., Chen, F., and Shen, S. (2007). Isolation and functional characterization of the JcERF gene, a putative AP2/EREBP domain-containing transcription factor, in the woody oil plant Jatropha curcas. Plant Mol. Biol. 63, 419-428. doi: 10.1007/s11103-006-9098-7

Tang, Y., Qin, S., Guo, Y., Chen, Y., Wu, P., Chen, Y., et al. (2016). Genomewide analysis of the AP2/ERF gene family in physic nut and overexpression of the JcERF011 gene in rice increased its sensitivity to salinity stress. PLoS ONE 11:e0150879. doi: 10.1371/journal.pone.0150879

Thompson, J. D., Gibson, T. J., Plewniak, F., Jeanmougin, F., and Higgins, D. G. (1997). The ClustalX windows interface: flexible strategies for multiple sequence alignment aided by quality analysis tools. Nucleic Acids Res. 25, 4876-4882. doi: 10.1093/nar/25.24.4876

Wang, X., Han, H., Yan, J., Fang, C., and Wei, W. (2015). A new AP2/ERF transcription factor from the oil plant Jatropha curcas confers salt and drought tolerance to transgenic tobacco. Appl. Biochem. Biotechnol. 176, 582-597. doi: 10.1007/s12010-015-1597-z

Wei, T., Deng, K., Liu, D., Gao, Y., Liu, Y., Yang, M., et al. (2016). Ectopic expression of DREB transcription factor, AtDREB1A, confers tolerance to drought in transgenic Salvia miltiorrhiza. Plant Cell Physiol. 57, 1593-1609. doi: $10.1093 / \mathrm{pcp} / \mathrm{pcw} 084$

Wessler, S. R. (2005). Homing into the origin of the AP2 DNA binding domain. Trends Plant Sci. 10, 54-56. doi: 10.1016/j.tplants.2004.12.007

Wu, T. M., Lin, W. R., Kao, C. H., and Hong, C. Y. (2015). Gene knockout of glutathione reductase 3 results in increased sensitivity to salt stress in rice. Plant Mol. Biol. 87, 555-564. doi: 10.1007/s11103-015-0290-5

Xiong, J., Zhang, L., Fu, G., Yang, Y., Zhu, C., and Tao, L. (2012). Drought-induced proline accumulation is uninvolved with increased nitric oxide, which alleviates drought stress by decreasing transpiration in rice. J. Plant Res. 125, 155-164. doi: 10.1007/s10265-011-0417-y

Yan, H., Jia, H., Chen, X., Hao, L., An, H., and Guo, X. (2014). The cotton WRKY transcription factor GhWRKY17 functions in drought and salt stress in transgenic Nicotiana benthamiana through ABA signalling and the modulation of reactive oxygen species production. Plant Cell Physiol. 55, 2060-2076. doi: $10.1093 / \mathrm{pcp} / \mathrm{pcu} 133$

Yang, H., Yu, C., Yan, J., Wang, X., Chen, F., Zhao, Y., et al. (2014). Overexpression of the Jatropha curcas JcERF1 gene coding an AP2/ERF-type transcription factor increases tolerance to salt in transgenic tobacco. Biochemistry 79, 1226-1236. doi: 10.1134/S0006297914110108

Yoshida, S., Forno, D. A., Cock, J. H., and Gomez, K. A. (1976). Laboratory Manual for Physiological Studies of Rice, 3rd Edn. Manila: International Rice Research Institute, 61-64.

Zhang, T., Zhang, D., Liu, Y., Luo, C., Zhou, Y., and Zhang, L. (2015). Overexpression of a NF-YB3 transcription factor from Picea wilsonii 
confers tolerance to salinity and drought stress in transformed Arabidopsis thaliana. Plant Physiol. Biochem. 94, 153-164. doi: 10.1016/j.plaphy.2015. 05.001

Zhang, X., Liu, X., Wu, L., Yu, G., Wang, X., and Ma, H. (2014). The SsDREB transcription factor from the Succulent halophyte suaeda salsa enhances abiotic stress tolerance in transgenic tobacco. Int. J. Genomics 2015, 1-13. doi: 10.1155/ 2015/875497

Zhang, X. X., Tang, Y. J., Ma, Q. B., Yang, C. Y., Mu, Y. H., Suo, H. C., et al. (2012). OsDREB2A, a rice transcription factor, significantly affects salt tolerance in transgenic soybean. PLoS ONE 8:e83011. doi: 10.1371/journal.pone. 0083011
Conflict of Interest Statement: The authors declare that the research was conducted in the absence of any commercial or financial relationships that could be construed as a potential conflict of interest.

Copyright () 2017 Tang, Liu, Zhang, Li, Xu, Zhang, Qi, Yu, Wang and Li. This is an open-access article distributed under the terms of the Creative Commons Attribution License (CC BY). The use, distribution or reproduction in other forums is permitted, provided the original author(s) or licensor are credited and that the original publication in this journal is cited, in accordance with accepted academic practice. No use, distribution or reproduction is permitted which does not comply with these terms. 